\title{
Atypical Presentation of Takayasu's Arteritis-A Case Report
}

\author{
V. Satish Kumar Rao ${ }^{1}$ Ponugoti Godhasiri \\ ${ }^{1}$ Department of Cardiology, Nizam's Institute of Medical Sciences, \\ Hyderabad, India \\ 2Department of Orthopaedics, Osmania Medical College, \\ Hyderabad, India \\ Ind J Car Dis Wom 2019;4:26-28
}

Address for correspondence V. Satish Kumar Rao, MD, DTCD, DM Resident, Department of Cardiology, Nizam's Institute of Medical Sciences, Hyderabad 500082, India (e-mail: drsatishrao09@gmail.com).

\begin{abstract}
Keywords

- pulseless

- arteritis

- atypical

- thrombosis

- stenosis

Takayasu's arteritis, known as "pulseless disease," is a chronic idiopathic inflammatory disease, which has a greater predilection for large vasculature in the body. Initially described in the1800s, this rare condition is more commonly seen in Asian women in 40 years age group. Herein, the authors report the case of a 36-year-old woman whose exertional claudication was the initial manifestation of active Takayasu's arteritis along with involvement of multiple peripheral arteries not involving ostial area of the vessels, instead affecting the proximal area and also presenting with thrombosis of right $\mathrm{CIA}$ (common iliac artery), which is an unusual presentation in case of Takayasu's arteritis without aneurysmal involvement.
\end{abstract}

\section{Introduction}

Takayasu's arteritis is an uncommon, systemic inflammation involving large vessels of unknown etiology that have a higher incidence in females of younger age group. It is defined as "granulomatous inflammation of the aorta and its major branches" by the Chapel Hill consensus conference on the nomenclature of systemic vasculitis. ${ }^{1}$ As it commonly involves brachiocephalic vessels, it is known as "pulseless disease" or "aortic arch syndrome."2 Takayasu's arteritis can present as isolated, atypical, and/or catastrophic disease. It can involve multiple or one major organ systems of the body. The condition is common in all parts of the world, although it seems to be more common in Asians. Aortic arch involvement is frequent in Japanese whereas abdominal aorta involvement is common in Indians with Takayasu's arteritis. ${ }^{3}$ In India, women-to-men ratios are as low as 1.6:1.1 The underlying etiology is chronic inflammation, and various etiologies have been proposed.

\section{Case History}

A 36-year-old woman, a known case of hypertension, came with 4-year history of intermittent claudication of Rutherford grade I in the right lower limb, which was progressively increasing in nature to Rutherford grade II. Previously the patient had dragging pain in right upper limb and one episode of giddiness not associated with loss of consciousness and palpitations. Over time, she had worsening right lower limb pain associated with coldness, pallor, numbness, and paresthesias, and gradually claudication distance was decreased to $100 \mathrm{~m}$. Recently she developed rest pain of lower limb and was in Rutherford grade III.

\section{Examination}

The patient was anxious with right cold lower limb with absent peripheral pulses. Pulse was felt well in the left upper limb with a pulse rate of 88 beats/min and blood pressure (BP) of 180/90 mm Hg. Right radial pulse was of low volume with BP of 110/80 mm Hg. Left carotid and left subclavian bruit was present. Precordium revealed normal findings. With the onset of symptoms before 40 years of age and presenting with prominent upper and lower limb ischemia in a female Takayasu's arteritis was diagnosed.

\section{Investigations}

Hemoglobin was $12.3 \mathrm{~g} \%$ with normal leukocyte count. Erythrocyte sedimentation rate (ESR) was elevated with $86 \mathrm{~mm}$ in the first hour along with positivity of C-reactive protein (CRP). Electrocardiographic (ECG) was normal. Two-dimensional (2D) echo showed no regional wall motion abnormality with good left ventricular function. Right lower limb arterial 
Doppler study demonstrated thrombosis of the right common iliac and external iliac arteries, which is an unusual finding in Takayasu's arteritis, except noted with aneurysmal involvement of vessels. Arterial Doppler of aortic arch and its branches showed reduced flow in left common carotid and no flow in right subclavian artery. Computed tomographic (CT) peripheral angiogram showed tight left common carotid artery stenosis and total occlusion of right subclavian artery along with thrombosis of the right common iliac artery (CIA).

Coronary angiogram showed normal epicardial coronaries. Carotid angiogram showed that the left common carotid artery had 70 to $80 \%$ stenosis and that the right common carotid artery was normal. Arch aortogram showed ostial total occlusion in the right subclavian artery (Fig. 1A, B). Lower limb arteriogram showed total occlusion in the right CIA, with reformation at the common femoral artery with distal occlusion at the level of the anterior and posterior tibial arteries (Figs. 1C, 2A). Left lower limb vessels were normal. A renal arteriogram showed right renal artery total cutoff at proximal portion but not at the ostium, whereas the left renal artery was normal (Fig. 2B). As the patient presented with rest pain in lower limb, vascular surgeon opinion was taken regarding thrombolysis and catheter-directed thrombolysis was done with urokinase for 24 hours. The patient improved after thrombolysis, and rest pain decreased.

\section{Discussion}

Takayasu's arteritis is a chronic inflammatory disease affecting large- and medium-sized arteries, mainly involving the aorta and its branches. ${ }^{4}$ Takayasu's arteritis is also known as "pulseless disease," "occlusive thromboarteriopathy," and

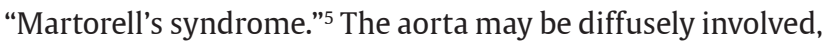
or any of its branches may be affected. Predominantly subclavian and carotid arteries ${ }^{6-9}$ are involved as in our case. Vessel stenosis is reported in more than $90 \%$ of patients as our index case, whereas an aneurysm is seen in nearly $25 \% 7,8$ of the cases. The presentation of the disease varies geographically. ${ }^{9-11}$ The site of arterial disease either in the arch or descending aorta determines its clinical presentation. The other visceral arteries such as the renal arteries and superior or inferior mesenteric arteries ${ }^{12}$ are involved. Classically all arterial stenosis affects ostium. In this case, subclavian involvement was ostial, but renal involvement was not ostial, and proximal involvement was seen.

Ishikawa graded Takayasu's arteritis based on the presence of four significant complications such as hypertension, retinopathy, aneurysm formation, and aortic regurgitation. ${ }^{13}$ Hypertension with Takayasu's arteritis is commonly renovascular, as in our case. It may be due to decreased elastic nature and severe stenosis of the aorta
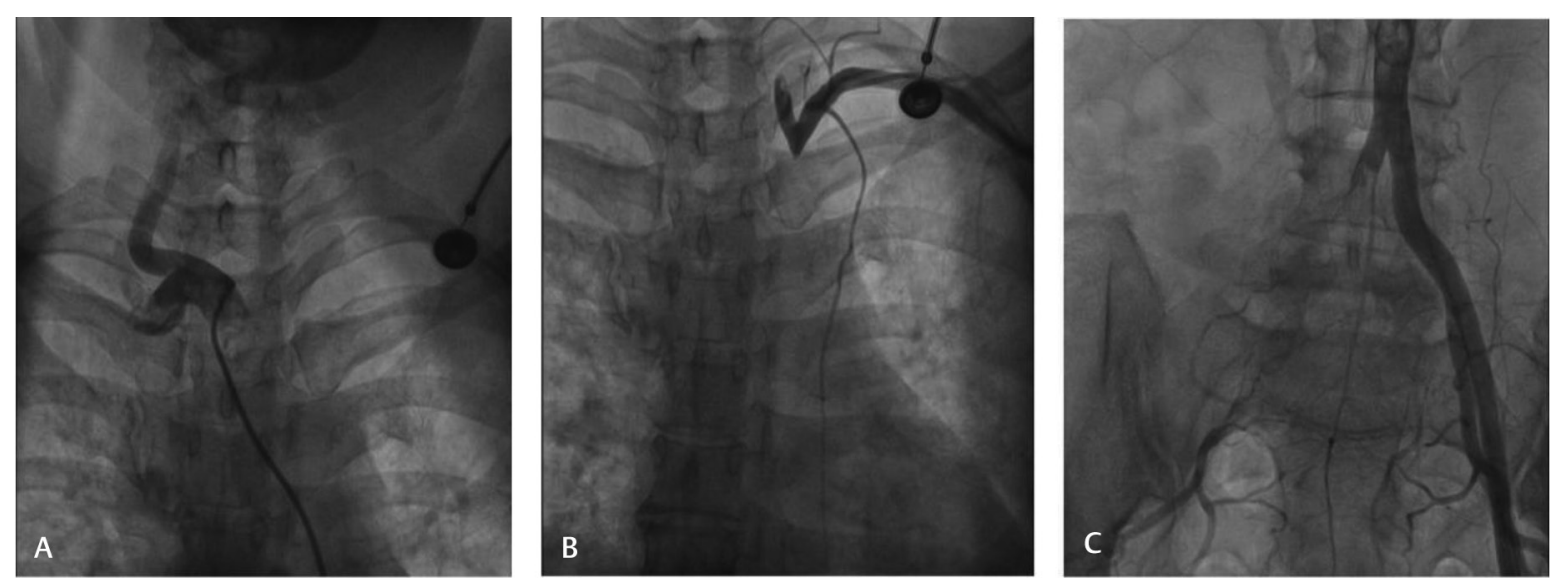

Fig. 1 Angiographic images. (A) Right subclavian artery showing no flow. (B) Left common carotid artery showing no flow. (C) Right common iliac artery showing thrombus containing lesion.
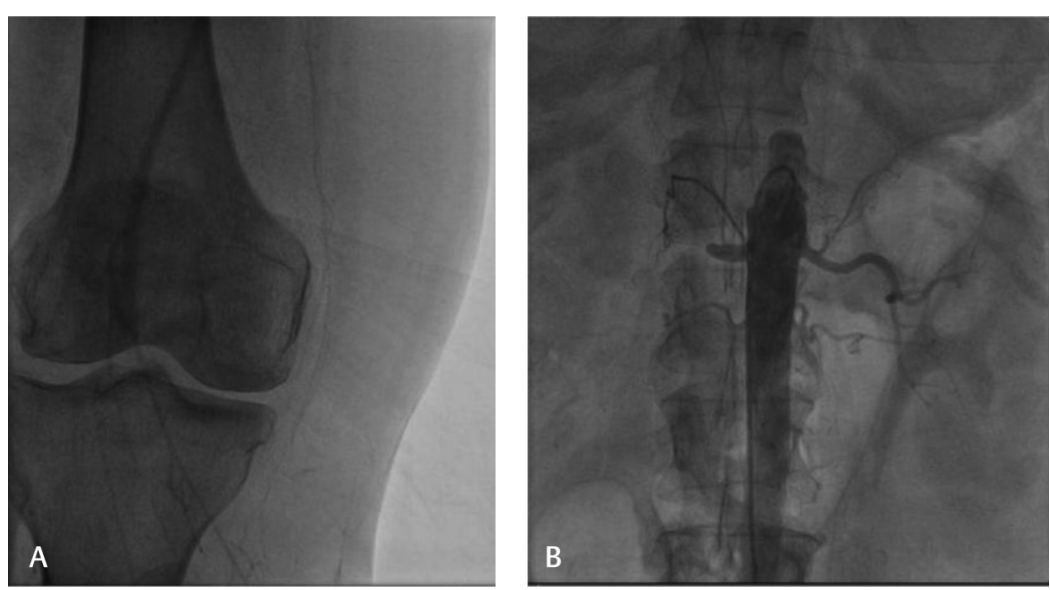

Fig. 2 Peripheral angiographic images. (A) Right popliteal artery no flow. (B) Right renal artery showing total cutoff and no flow. 
and major arteries and impaired baroreceptors regulation of carotid and aortic sinus. ${ }^{14}$ Recording of BP and palpation of all pulses is essential. Noninvasive BP recording by oscillometric method and pulse oximetry give easy and reliable BP recordings patients with in pulseless extremities. ${ }^{11}$ Descending aorta involvement especially around the renal arteries, which is called as "middle aortic syndrome," is a well-described entity in Takayasu's arteritis, but the involvement of the iliac artery and femoral artery is relatively rare. Mandalam et al reported 20 cases of common femoral artery diseases without contiguous involvement of the external iliac and superficial femoral arteries. Among them two were of Takayasu's arteritis. ${ }^{15}$ Pistorius et al reported a case of Takayasu's arteritis with aortoiliac disease thrombus in a 30-year-old female patient because of its rarity. ${ }^{16} \mathrm{~A}$ series of Takayasu's arteritis by Chung et al showed $15 \%$ involvement of the right or left iliac arteries, of which either stenosis or wall thickening on CT was demonstrated, but aneurysmal involvement was not seen. ${ }^{17}$

Similarly Arnaud et al, in 82 patients with Takayasu's arteritis, demonstrated 15 patients with iliac artery involvement. ${ }^{18}$ Aneurysm formation, though rarer than stenosis, is an important cause of death when it ruptures. This variety of Takayasu's arteritis was reported to be associated with thrombus formation. However, our case is a purely stenotic variety with thrombosis of the CIA. Similar to our case, Ostertag-Hill et al reported a rare case of Takayasu's arteritis with iliac stenosis superadded with thrombus. ${ }^{19,20}$ Treatment modalities of Takayasu's arteritis depend on the activity of disease along with the opening of occluded arteries. Corticosteroids effectively suppress inflammation in the active phase, and good results are seen with immunosuppressive and cytotoxic agents. ${ }^{21}$ Surgical intervention is required to reduce symptoms due to arterial obstruction, and percutaneous angioplasty with stenting gives good outcomes. These interventions are best done in patients when acute inflammation is under control.

\section{Conclusion}

This case of Takayasu's arteritis is reported due to the presence of few rare associations such as proximal renal artery involvement instead of ostial involvement and also iliac artery with thrombus, which is also uncommon except in aneurysmal involvement. However, in our case, there were no aneurysms, but thrombus was seen with a stenotic lesion.

\section{Conflict of Interest}

None.

\section{References}

1 Jennette JC, Falk RJ, Andrassy K, et al. Nomenclature of systemic vasculitides. Proposal of an international consensus conference. Arthritis Rheum 1994;37(2):187-192
2 Elefteriades JA, Olin JW, Halperin JL. Diseases of the aorta. In: Fuster V, Walsh RA, Harrington RA, eds. Hurst's the Heart. 13th ed. New York, NY: McGraw-Hill; 2011:2285-2286

3 Numano F, Kobayashi Y. Takayasu arteritis-beyond pulselessness. Intern Med 1999;38(3):226-232

4 Satsangi DK. Surgical experience with aortoarteritis in India. Indian J Thoracic Cardiovasc Surg 2007;23:110-115

5 Lupi-Herrere E, Sánchez-Torres G, Marcushamer J, et al. Takayasu arteritis. Clinical studyof107cases. Am Heart J 1997;93:94-103

6 Jain S, Kumari S, Ganguly NK, Sharma BK. Current status of Takayasu arteritis in India. Int J Cardiol 1996;54(Suppl): S111-S116

7 Kerr GS, Hallahan CW, Giordano J, et al. Takayasu arteritis. Ann Intern Med 1994;120(11):919-929

8 Mwipatayi BP, Jeffery PC, Beningfield SJ, et al. Takayasu arteritis: clinical features and management: report of 272 cases. ANZ J Surg 2005;75(3):110-117

9 Ishikawa K, Maetani S. Long-term outcome for 120 Japanese patients with Takayasu's disease. Clinical and statistical analyses of related prognostic factors. Circulation 1994; 90(4):1855-1860

10 Sharma BK, Sagar S, Singh AP, Suri S. Takayasu arteritis in India. Heart Vessels Suppl 1992;7:37-43

11 Park MC, Lee SW, Park YB, Chung NS, Lee SK. Clinical characteristics and outcomes of Takayasu's arteritis: analysis of 108 patients using standardized criteria for diagnosis, activity assessment, and angiographic classification. Scand J Rheumatol 2005;34(4):284-292

12 Moriwaki R, Noda M, Yajima M, Sharma BK, Numano F. Clinical manifestations of Takayasu arteritis in India and Japan-new classification of angiographic findings. Angiology 1997;48(5):369-379

13 Stoelting RK, Dierdorf SF. Anesthesia and Co-Existing Disease. 4th ed. New York, NY: Churchill Livingstone; 2002

14 Ishikawa K. Diagnostic approach and proposed criteria for the clinical diagnosis of Takayasu's arteriopathy. J Am Coll Cardiol 1988;12(4):964-972

15 Mandalam KR, Rao VR, Sandhyamani S, et al. Focal occlusive disease of the common femoral artery: a report of 20 cases. Cardiovasc Surg 1994;2(4):498-502

16 Pistorius MA, Jego P, Sagan C, Noel S, Dupas B, Planchon B. [Arterial embolic manifestations in the legs revealing isolated aorto-iliac Takayasu's disease] [in French] J Mal Vasc 1993;18(4):331-335

17 Chung JW, Kim HC, Choi YH, Kim SJ, Lee W, Park JH. Patterns of aortic involvement in Takayasu arteritis and its clinical implications: evaluation with spiral computed tomography angiography. J Vasc Surg 2007;45(5):906-914

18 Arnaud L, Haroche J, Toledano D, et al. Cluster analysis of arterial involvement in Takayasu arteritis reveals symmetric extension of the lesions in paired arterial beds. Arthritis Rheum 2011;63(4):1136-1140

19 Ostertag-Hill CA, Abdo AK, Alexander JQ, Skeik N. Unique case of Takayasu arteritis with severe distal aortic stenosis and iliac thrombosis. Ann Vasc Surg 2016;32:128.e7-128.e13

20 Box C, Dhillon V, McKay N, Amft N, Hauser B. Takayasu arteritis: a case series of unusual complications and comorbidities. Rheumatology 2018;57(3)

21 Arend WP, Michel BA, Bloch DA, et al. The American College of Rheumatology 1990 criteria for the classification of Takayasu arteritis. Arthritis Rheum 1990;33(8):1129-1134 